\title{
APPROCHE CONCEPTUELLE DE LA MODÉLISATION DE LA DYNAMIQUE DU STOCK D'ANGUILLES DANS UN BASSIN VERSANT : INTÉRÊT ET ADAPTATION DU MODĖLE DE RENDEMENT PAR RECRUE.
}

\author{
D. GASCUEL et G. FONTENELLE
}

Unité Halieutique / D. E. E. R. N.

Ecole Nationale Supérieure Agronomique, 65 Rue de St Brieuc, 35042 RENNES Cedex,

(France).

\begin{abstract}
RÉSUMÉ
A partir de l'approche structurale utilisée en halieutique pour des stocks exploités de poissons, une adaptation à l'Anguille au sein d'un bassin versant est présentée. Cette approche conceptuelle essaie de considérer toutes les particularités de cette espèce amphihaline exploitée à tous ses stades biologiques pour estimer les paramètres conduisant à l'élaboration d'un modèle. Les résultats que l'on peut attendre d'un tel modèle sont mis en évidence. stocks.

Mots-clés : dynamique de populations, Anguilla anguilla, bassin versant, gestion des

\section{A CONCEPTUAL APPROACH OF MODELLING AN EEL STOCK DYNAMICS WITHIN A WATERSHED : INTEREST AND ADAPTATION OF A YIELD PER RECRUIT MODEL.}

\begin{abstract}
From the structural approach used in fisheries science and applied to exploited stocks of marine fish, an adaptation to the Eel within a watershed is shown. This conceptual approach tries to consider all features of this amphihaline and all stages exploited species to estimate parameters involved in making a model. The expected results from such a model are emphasized.
\end{abstract}

Key-words : population dynamics, Anguilla anguilla, watershed, stock management.

\section{INTRODUCTION}

L'Anguille européenne (Anguilla anguilla L.) constitue une ressource biologique, économique et sociale commune à tous les pays de la façade Atlantique ouest. Elle fait l'objet d'une exploitation et d'un marché international très actif. Tous les indices disponibles indiquent à l'heure actuelle une diminution de l'abondance de cette ressource (ANONYME, 1984 a et b ; ANONYME, 1988) sans que les causes, sans doute multiples, puissent en être clairement déterminées.

Face à cette situation, la recherche d'une gestion globale du stock, à l'échelle de son aire de distribution (Europe et Afrique du Nord), apparaît non seulement très difficile à mettre en œuvre mais surtout largement illusoire quant à son efficacité (GASCUEL, 1987 b). En particulier, il est hautement improbable qu'existe une relation identifiable entre le nombre de géniteurs quittant le domaine continental au stade "anguille argentée" et le nombre de recrues qui en est issu quelques années plus tard, arrivant sur les côtes au stade civelle.

Une autre approche est en revanche possible. S'inspirant du modèle de rendement par recrue (RICKER, 1945 et 1958 ; PAULICK et BAYLIFF, 1967) communément utilisé en halieutique marine, elle consiste à rechercher la meilleure utilisation possible du recrutement disponible quelle que soit par ailleurs l'importance de ce recrutement. Cette démarche, et plus généralement ce qu'on appelle l'approche structurale, présente en particulier un intérêt majeur : elle permet d'envisager une gestion de la ressource à l'échelle d'un bassin versant pris dans son entier (partie estuarienne et fluviale). 
Cependant, le modèle de rendement par recrue habituellement utilisé ne peut être directement transposé au cas de l'Anguille. Des adaptations doivent être envisagées en raison des particularités de la biologie de l'espèce et de son exploitation. C'est à cette adaptation du modèle de rendement par recrue au cas de l'Anguille, et donc plus généralement à un schéma d'approche conceptuelle de la dynamique du stock dans un bassin versant, qu'est consacré cet article. II prolonge ainsi la réflexion engagée au sein du Groupe National "Anguille" (ANONYME, 1984 $a$ et b), en précisant les méthodologies de modélisation à mettre en oeuvre en vue d'une gestion raisonnée de l'unité de stock présente dans un même bassin versant.

\section{RAPPELS : LA NOTION DE RENDEMENT PAR RECRUE}

Fondamentalement, l'utilisation d'un modèle de rendement par recrue est justifiée par l'idée suivante : le recrutement d'un stock (nombre de poissons entrant chaque année dans une pêcherie) ne dépend pas de l'abondance des géniteurs dont il provient ; il est par conséquent indépendant de l'intensité et des caractéristiques de l'exploitation de ce stock. Cette idée peut paraître paradoxale ; elle est cependant très largement confortée par l'ensemble des résultats scientifiques établis en halieutique marine (CUSHING, 1973 ; LAUREC, 1990). Elle s'explique bien lorsqu'on connaît, d'une part l'extraordinaire fécondité de la plupart des ressources vivantes aquatiques (comparativement à celles des ressources terrestres) et d'autre part la très grande mortalité naturelle affectant la "phase pré-recrutée", notamment les stades larvaires. On conçoit dès lors aisément que le nombre de recrues dépend bien plus des variations des taux de mortalité avant recrutement, en liaison notamment avec les variations des facteurs écologiques (biotiques et abiotiques), que de l'abondance des géniteurs. A de rares cas particuliers près, cette idée de base ne souffre guère qu'une seule exception d'envergure : lorsque l'exploitation ou plus généralement l'action anthropique se traduit par une diminution considérable de l'abondance des géniteurs. Si on poursuit le raisonnement jusqu'à sa limite, il est en effet évident qu'un stock de géniteur nul, produira un recrutement nul. De manière empirique (LAUREC, 1990), on considère cependant que l'effet dépresseur d'une baisse de l'abondance des géniteurs sur le recrutement n'est, en général, susceptible de se faire sentir que pour des abondances inférieures à $5 \%$ ou $10 \%$ de celle d'un stock à l'état vierge (absence d'exploitation).

Dans le cas de l'Anguille, la mise en évidence d'une relation entre le nombre d'anguilles argentées quittant le littoral atlantique et le nombre de civelles recrutées quelques années plus tard apparaît ainsi hautement hypothétique. La fécondité de cette espèce est en effet considérable et le taux de mortalité des larves très élevé ; il dépasserait 99,5\% (TESCH, 1980). En outre, même si des indices de baisse de l'abondance du stock existent, l'Anguille reste encore une espèce très abondante dans la plupart des pays européens.

Enfin, l'Anguille est une espèce migratrice dont les juvéniles au stade civelle se répartissent dans tous les bassins versants de l'aire de distribution, indépendamment, selon toute vraisemblance, de la provenance de leurs géniteurs. Autrement dit, toute politique de gestion du stock dont l'objectif serait de protéger le potentiel reproducteur, en vue d'accroître le recrutement, paraît vouée à l'échec, tant d'un point de vue théorique que pratique. Pour des résultats, à terme hypothétiques, il faudrait, en effet, que des mesures de gestion significatives soient prises à l'échelle des différents pays, dans les différents milieux habités par l'Anguille, et pour les différents stades biologiques exploités...

Si on admet cette absence de relation entre stock de géniteurs et recrutement, le seul problème est d'utiliser au mieux le recrutement disponible. La démarche la plus courante consiste alors à rechercher une maximisation des captures. Le dilemme est simple :

— faut-il pêcher les animaux jeunes, c'est-à-dire lorsqu'ils sont nombreux mais de faible poids individuel?

- ou faut-il adopter des mesures de limitation de l'effort et de réglementation des engins de pêche, conduisant à la capture d'animaux en moyenne plus âgés, moins nombreux (mortalité naturelle), mais plus gros (croissance pondérale) ?

Ce bilan entre Mortalité et Croissance dépend des caractéristiques biologiques de l'espèce et des caractéristiques de la pêcherie (Régime d'exploitation). Sa quantification est l'objet du modèle de rendement par recrue (RICKER, 1945 et 1958 ; PAULICK et BAYLIFF, 1967). L'absence de relation entre stock de géniteurs et recrutement est, par ailleurs, une justification 
suffisante mais non nécessaire à l'utilisation de ce modèle. En effet, de la même manière, le modèle de rendement par recrue peut être utilisé pour estimer une production de géniteurs en fonction des caractéristiques de l'exploitation affectant les différents stades biologiques.

Dans le cas de l'Anguille, on s'intéressera aussi aux deux grandeurs "Captures" et "Production" de géniteurs. Pour chaque cohorte annuelle, recrutée au stade civelle dans un bassin versant donné (estuaire et zone fluviale), on cumulera les captures effectuées sur cette cohorte sur l'ensemble de sa vie (stades civelle, anguille jaune et anguille argentée) ; on estimera la production de géniteurs, en nombre ou en biomasse, quittant ce bassin versant au stade anguille argentée, pour aller se reproduire en Mer des Sargasses.

L'effectif au recrutement restant inconnu, ces résultats seront exprimés en valeurs moyennes par recrue ou pour 100 recrues. Le modèle conduit ainsi à une formulation mathématique qui indique, en fonction des paramètres caractéristiques de l'exploitation, l'effectif et la biomasse : des captures, des animaux morts naturellement et des géniteurs échappant à ces deux mortalités (ces grandeurs étant rapportées à 100 civelles ou à $100 \mathrm{~g}$ de civelles recrutées).

\section{L'APPROCHE STRUCTURALE : LA DÉMARCHE USUELLE UTILISÉE EN HALIEUTIQUE MARINE.}

Le modèle de rendement par recrue s'inscrit en fait dans un ensemble d'approche méthodologique plus large qui constitue la modélisation structurée par âge ou approche structurale (Figure 1) dont on en rappelera ici brièvement le principe.

L'analyse des cohortes (GULLAND, 1965) constitue classiquement la première étape. A partir d'une matrice des captures par âge (les lignes correspondent aux différents âges et les colonnes aux années pour lesquelles les statistiques de pêche sont disponibles) et d'une matrice similaire des taux instantanés de mortalité naturelle, cette méthode d'analyse permet de construire d'une part, la matrice des effectifs du stock par âge et par année et, d'autre part, la matrice correspondante des taux instantanés de mortalité par pêche. Pour être plus exact, il faut préciser que le système d'équations ainsi défini comporte cependant une indétermination pour chaque cohorte considérée. Cette difficulté peut être résolue, soit par l'introduction d'un paramètre d'entrée supplémentaire (généralement le taux d'exploitation terminal qui équivaut au rapport entre mortalité par pêche et mortalité totale pour le groupe d'âge le plus âgé), soit par l'utilisation de procédures complémentaires telles que le "tuning" (POPE et SHEPHERD, 1985) ; l'analyse est alors calibrée en fonction de données d'effort de pêche ou d'indices d'abondance.

L'analyse des cohortes est, en premier lieu, un outil extrêmement puissant d'investigation de l'histoire passée et récente du stock. Elle peut, de ce point de vue, être considérée comme un modèle explicatif. Pour des raisons de convergence de l'analyse, qu'il serait trop long de détailler ici (1) l'image obtenue est d'autant plus fiable qu'elle concerne un passé éloigné. A l'inverse, les résultats obtenus sur la période récente sont plus sensibles à l'estimation des paramètres d'entrée du modèle. Ceci est particulièrement vrai pour les stocks faiblement exploités.

Le second intérêt majeur de l'analyse des cohortes réside dans l'estimation des paramètres "Effectif du stock" et "Mortalité par pêche". Ces paramètres, auxquels il convient d'ajouter une estimation des poids moyens par âge, constituent à leur tour des variables d'entrée pour les modèles de prédictions à court et à long terme.

Le modèle de rendement par recrue permet ainsi de calculer la biomasse des captures effectuées en moyenne pour chaque recrue entrant dans la pêcherie. Ce rendement peut être estimé sous l'hypothèse d'un régime d'exploitation constant en considérant que les mortalités par pêche seront à l'avenir identiques à celles estimées pour la dernière année (ou la moyenne pour les dernières années) prise en compte dans l'analyse des cohortes. De même, toute modification du régime d'exploitation peut être simulée, en modifiant judicieusement ces mortalités par pêche. Une augmentation globale de l'effort de pêche sera par exemple traduite en multipliant les mortalités par pêche à chaque âge par un coefficient correspondant à cette augmentation. 
DEMARCHE USUELLE DE LA MODELISATION EN HALIEUTIQUE PAR L'APPROCHE STRUCTURALE

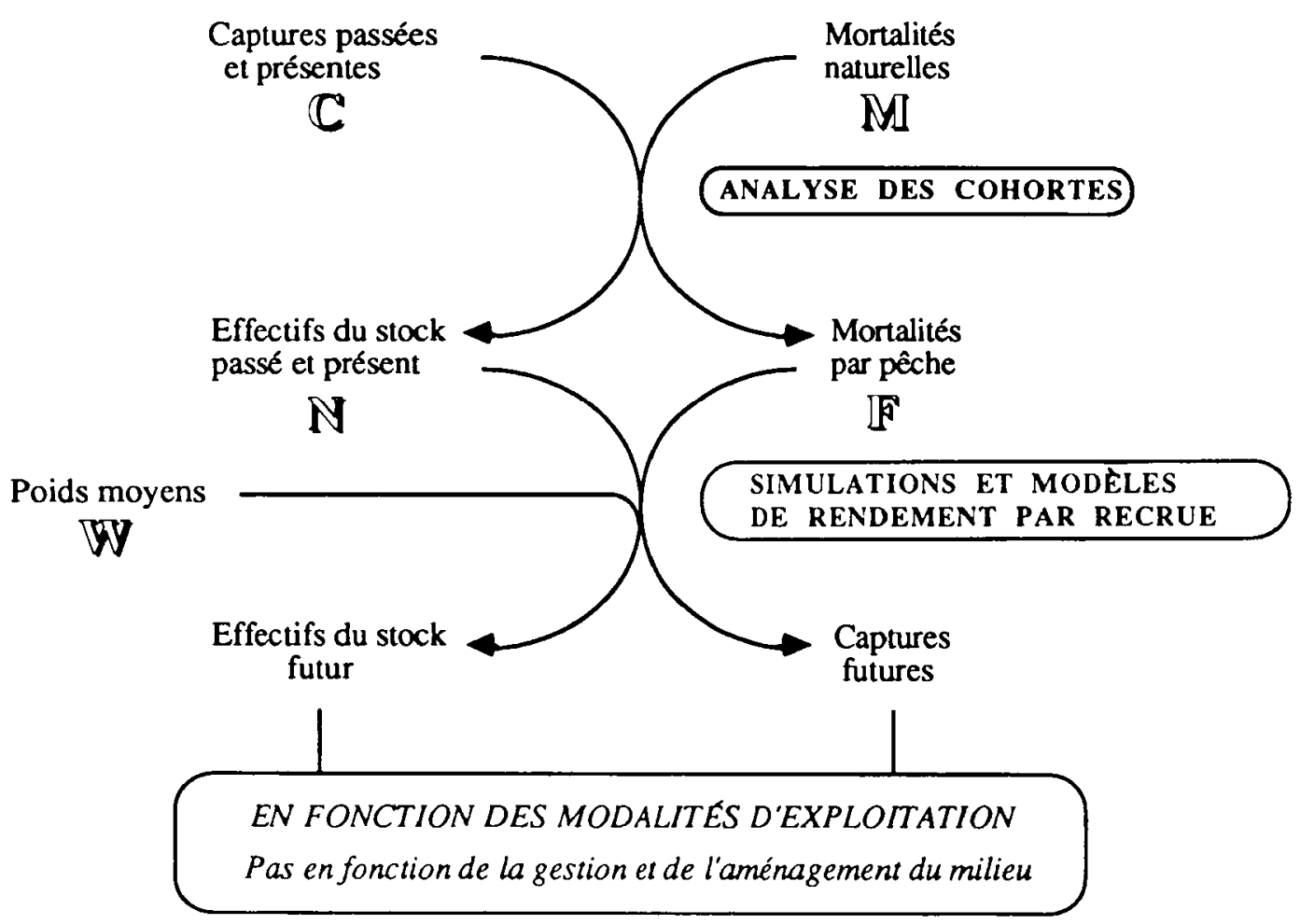

Figure 1 : Schéma illustrant la démarche usuelle de la modélisation en halieutique marine : l'approche structurale. Cette démarche peut éventuellement être appliquée à la fraction estuarienne du stock d'anguilles jaunes, mais non à la fraction dulçaquicole.

Figure 1 : Flow chart of usual modelling in marine fisheries science : the structural approach. This approach may be applied to the estuarine part of yellow eels stocks, but not to the freshwater part.

Une augmentation de maillage sera quant à elle simulée, en attribuant des valeurs nulles, aux mortalités par pêche correspondant aux âges jeunes épargnés par le nouveau maillage.

Le modèle de rendement par recrue débouche sur une prédiction à long terme : les estimations effectuées concernent les captures obtenues sur toute la durée de vie de la cohorte suivie du recrutement jusqu'au décès du dernier individu. Ce diagnostic peut être complété par des précisions à court terme grâce aux techniques de simulations ${ }^{(2)}$. La principale limitation de ces techniques réside dans la nécessité d'introduire une estimation du recrutement futur, pour chacune des années simulées. Lorsqu'on simule des années successives, on aboutit ainsi rapidement à une situation dans laquelle le diagnostic ne dépend plus que de ces recrutements introduits et ne présente donc plus aucune valeur prédictive.

(2) Pour plus de détails, se reporter à WALTERS (1969), LAUREC et LE GUEN (1981), BRETHES et O'BOYLE (1990). 
Enfin, rappelons que le modèle de rendement par recrue peut être complété par une estimation de la production moyenne de géniteurs par recrue. L'approche structurale constitue ainsi un outil de prédiction extrêmement puissant, tant de l'état futur du stock que des captures, en fonction des modalités d'exploitation qui seront appliquées.

Ces méthodes sont couramment utilisées en halieutique marine. Elles fournissent dans ce cas des diagnostics d'autant plus fiables que l'on s'intéresse à des stocks exploités de manière intense (POPE, 1972 ; LAUREC, 1990). Leur utilisation pour la définition de politiques de gestion des pêches s'avère alors extrêmement précieuse même si d'autres éléments (économiques, sociaux ou ayant trait aux variations de l'environnement) doivent également être pris en compte.

\section{QUELQUES PARTICULARITÉS DE L'ANGUILLE ET DE SON EXPLOITATION}

La première particularité biologique de l'Anguille à prendre en compte est bien évidemment son caractère migratoire. Celui-ci a plusieurs conséquences.

En premier lieu, et c'est un aspect positif en tant que source de simplicité et de rigueur, l'unité de stock prise en compte pour la gestion peut être définie très clairement. C'est l'ensemble des animaux peuplant le réseau hydrographique d'un bassin versant fluvial, y compris la zone estuarienne. La limite "aval" du stock ainsi défini correspond à la zone marine; les études concernant les peuplements estuariens d'anguilles montrent en effet une décroissance des densités à l'aval des estuaires (GASCUEL et FONTENELLE, 1989 et 1990) et une quasi absence dans le domaine maritime. Si quelques déplacements d'anguilles jaunes semblent avoir été mis en évidence d'un estuaire à un autre, il n'en demeure pas moins que ce phénomène apparaît quantitativement très limité. Les échanges entre stocks sont ainsi tout à fait négligeables (en tout cas très inférieurs à ce qu'ils peuvent être dans la plupart des stocks marins faisant l'objet d'une modélisation).

De même, le recrutement peut ici être défini très clairement. ll correspond à l'arrivée des civelles dans la zone estuarienne, dont la majeure partie en période hivernale (GUERAULT et al., 1991). II permet de définir un âge relatif, compté à partir de cette arrivée, indépendamment de la durée de la phase marine précédente. En outre, l'existence dans la plupart des estuaires du littoral atlantique français d'une exploitation commerciale de civelles permet de disposer d'indices d'abondance du recrutement (GUÉRAULT et al., 1986 ; GASCUEL, 1987 b ; DESAUNAY et al., 1987 ; GUÉRAULT et al., 1987 ; ELIE et al., 1989 ; GUÉRAULT et DESAUNAY, 1989 ; BEN ABDALLAH, 1991) qui pourront s'avérer utiles dans des analyses sur une longue période.

Le caractère migratoire de l'espèce conduit ensuite à distinguer deux fractions de stocks différentes et pour une part indépendantes (Figure 2). Une partie des civelles arrivant en estuaire se sédentarise en effet sur place et accomplit dans ce milieu l'ensemble de sa phase de croissance et de différenciation sexuelle jusqu'au stade "anguille argentée". Le peuplement de la zone fluviale proprement dit est assuré, quant à lui, d'une part par des civelles qui arrivent dans l'estuaire en hiver et poursuivent leur migration vers l'amont au printemps suivant (ELIE et RIGAUD, 1984 ; LEGAULT, 1986 ; GASCUEL, 1987 a et b ; LEGAULT et PORCHER, 1989), et d'autre part par des anguilles jaunes qui, après une sédentarisation temporaire dans l'estuaire, reprennent leur migration à un stade ultérieur (LEGAULT, 1986 ; GASCUEL et FONTENELLE, 1989 et 1990). Les études les plus récentes sur la structure des otolithes des anguilles jaunes et argentées (MOUNAIX, 1991, 1992 a et b ; PANFILI et al., 1992) confirment notamment la relative indépendance de ces deux fractions de stocks. En particulier, le peuplement estuarien correspondrait bien à des animaux sédentarisés sur place et non à l'incursion temporaire d'animaux en provenance du domaine fluvial.

Enfin, l'existence d'une migration anadrome de reproduction chez l'anguille se traduit par le fait que la production de géniteurs correspond à un échappement du système. Du point de vue de la modélisation, la dévalaison peut ainsi être traitée de la même manière qu'une mortalité, par l'estimation de taux instantanés de dévalaison. Ceci a naturellement des conséquences en matière de méthode d'estimation des paramètres d'entrée du modèle. Ceci a également des conséquences quant à la manière dont la gestion des stocks devra être envisagée. En effet, en raison du cycle de vie de l'Anguille, toute exploitation de l'espèce ne peut intervenir que sur des stades juvéniles et prépubères avant reproduction. L'impact de ces exploitations sur la production de géniteurs dépend en définitive des taux d'exploitation de chaque stade. 


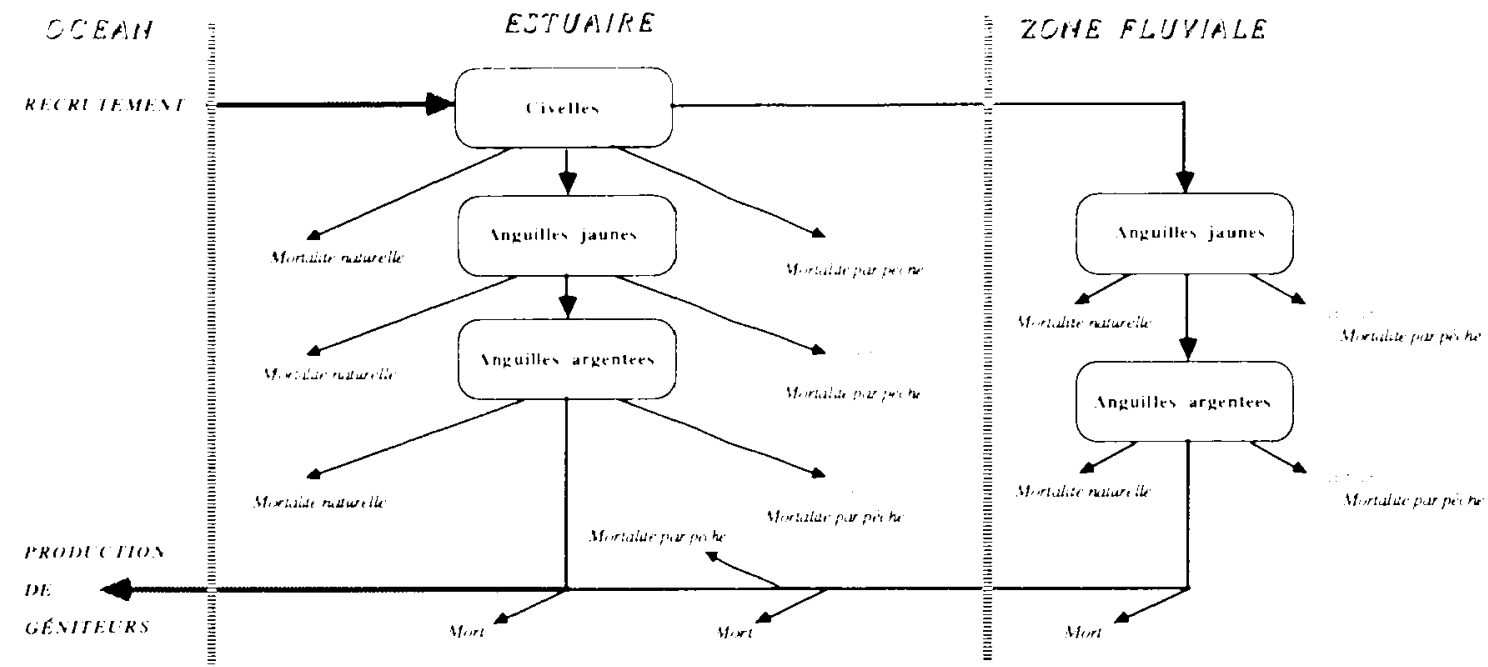

Figure 2 : Schéma simplifié illustrant l'évolution des effectifs au cours du cycle de l'anguille selon les milieux et les stades biologiques : deux fractions doivent être distinguées.

Figure 2 : Simplified flow chart displaying the evolution in numbers of the eel during its life cycle with respect to habitats and biological stages : two parts must be separated.

On aborde ici une autre caractéristique essentielle de la biologie de l'Anguille qui est l'existence de trois stades biologiques distincts au cours de la vie continentale. Les stades civelle, anguille jaune et anguille argentée présentent des différences non seulement morphologiques et anatomiques, mais aussi métaboliques, physiologiques et comportementales. Ces différences se traduisent, selon toute vraisemblance, par des variations de la mortalité naturelle. De plus, si la transformation civelle / anguille jaune intervient sensiblement au même âge chez tous les individus, il n'en va pas de même pour la métamorphose anguille jaune / anguille argentée. L'âge de métamorphose dépend ici des taux de croissance (ROSSI et COLOMBO, 1979 ; VOLLESTAD, 1989) et de la différenciation sexuelle (COLOMBO et al., 1984).

Au plan de la modélisation, cette métamorphose pourra elle aussi être traitée comme une mortalité apparente des anguilles jaunes par l'intermédiaire de 'taux d'argentures". L'avalaison succédant rapidement à l'argenture, ces taux pourront être considérés directement comme la somme de trois taux, correspondant respectivement à la mortalité naturelle, à la mortalité par pêche et à l'échappement vers l'océan des anguilles argentées.

La modélisation du stade Anguille jaune s'attachera au suivi d'une cohorte d'âge en âge et d'année en année ; chaque année, tout groupe d'âge sera sujet à une mortalité naturelle, une mortalité par pêche et une mortalité apparente "par argenture". La fraction échappant à ces phénomènes constituera l'année suivante le groupe d'âge supérieur.

La modélisation du stade Anguille argentée ne concernera qu'une seule année : chaque groupe d'âge "recruté" dans ce stade par le phénomène d'argenture disparaît en effet au cours de l'année soit par mortalité naturelle ou par pêche, soit par échappement vers l'océan.

Enfin, et c'est sans doute l'essentiel, la succession des différents stades biologiques donne lieu à des méthodes de pêche différentes qui sont mises en oeuvre par plusieurs catégories de pêcheurs. On a ainsi affaire à des pêcheries séquentielles intéressant successivement plusieurs groupes sociaux. Plus encore que pour tout autre type de stock, la double distinction, entre fraction estuarienne et fluviale d'une part, et entre stades biologiques d'autre part, rend tout à fait illusoire la recherche d'une gestion dite "rationnelle" du stock. L'utilisation d'un modèle de recrutement par recrue et la recherche d'une simple maximisation des captures conduiraient en effet inexorablement à ne conserver qu'un seul type d'exploitation. Il est fort douteux qu'une telle évolution soit "rationnelle" ou "optimale" ne serait-ce que du point de vue de la gestion des ressources humaines que constituent les pêcheurs eux-mêmes... 
Cette observation ne condamne pas l'approche modélisatrice à l'impuissance, bien au contraire, puisque cette approche doit notamment permettre de connaître et simuler les interactions entre pêcheries. Mais il est clair qu'on s'éloigne de la vision très réductrice d'une "optimisation du rendement par recrue". Ceci est encore plus vrai, si on prend également en compte la production de géniteurs par recrue, comme nous l'avons évoqué plus haut.

\section{ADAPTATION DES MODÈLES AU CAS DE L'ANGUILLE. GESTION DES PÊCHERIES ET GESTION DU MILIEU.}

Si on tient compte des remarques précédentes, la démarche classiquement utilisée en halieutique marine peut raisonnablement être envisagée en ce qui concerne la fraction estuarienne du stock. Elle doit, à terme, déboucher sur une expression des rendements par recrues (en civelle, anguille jaune et anguille argentée) ainsi que sur une expression de la production de géniteurs par recrues en fonction des caractéristiques des trois pêcheries correspondant au trois stades biologiques. Une partie de cette approche a déjà été réalisée en Norvège (VOLLESTAD et JONSSON, 1988)

Pour la fraction fluviale du stock, la démarche classique ne peut en revanche pas être directement transposée pour deux raisons principales :

En premier lieu, l'analyse des cohortes est une méthode d'analyse sensible à l'estimation des captures et qui requiert donc des statistiques de pêche fiables. De plus, les propriétés de convergence de l'analyse, évoquées plus haut, dépendent directement de l'intensité de l'exploitation du stock considéré. En d'autres termes, si les résultats obtenus sont généralement fiables dans le cas de stocks fortement exploités, ils peuvent être très douteux dans le cas d'exploitations de faible intensité.

En ce qui concerne l'Anguille, les captures effectuées dans les cours d'eau se jetant dans l'Océan atlantique et la Manche sont pour l'essentiel le fait de pêcheurs amateurs et /ou de pêcheurs professionnels (BABIN, 1983). Les statistiques de pêche sont presque totalement inconnues ; leur estimation avec un minimum de précision apparaît très complexe et leur niveau global est sans doute faible comparativement à la "productivité" du stock. Il y a donc tout lieu de penser que l'analyse des cohortes serait ici une méthode peu performante quant à la fiabilité des résultats obtenus.

La seconde difficulté se rapporte autant à des aspects méthodologiques qu'à la nature même des objectifs poursuivis par la modélisation.

En milieu marin, la mortalité naturelle est très généralement considérée constante d'année en année. Cette hypothèse paraît raisonnable compte tenu de la relative constance des caractéristiques du milieu. Plus fondamentalement, elle se justifie en considérant les fluctuations éventuelles de ce paramètre comme exclusivement liées aux variations erratiques de l'environnement, donc totalement indépendantes de l'action anthropique.

Le milieu dulçaquicole est, quant à lui, sujet à une très grande variabilité temporelle, liée à des facteurs naturels, mais aussi à des facteurs humains. Une pollution permanente ou accidentelle, la construction d'un ouvrage hydraulique, un recalibrage de rivière risquent par exemple de modifier radicalement le milieu et de se traduire par des variations d'abondance et de mortalité naturelle. De surcroît, ces exemples montrent à l'évidence qu'à la variabilité temporelle est associée une variabilité spatiale. L'hypothèse d'une mortalité naturelle constante dans le temps et unique à l'échelle de l'ensemble du bassin versant n'apparaît donc ni justifiable ni même souhaitable.

En milieu marin, on s'intéresse essentiellement aux pêcheries parce qu'elles représentent la principale source de variabilité des stocks et surtout parce qu'elles constituent le seul élément de gestion sur lequel il est possible d'intervenir. En milieu dulçaquicole, on doit associer une gestion du milieu à la gestion des pêcheries en visant notamment à une minimisation de la mortalité naturelle. Ainsi, ce paramètre ne peut être ni estimé, ni utilisé comme dans la démarche classique.

Fort heureusement, les difficultés d'estimation des paramètres "Captures" d'une part et "Mortalité naturelle" d'autre part, peuvent en quelque sorte être "compensées" par l'accès à une autre donnée: "l'Abondance" réelle du stock par groupe d'âge. 
En milieu marin, nul halieute n'envisage une quantification directe du nombre de poissons présents dans l'eau pour l'ensemble du stock et par groupe d'âge ; la donnée est méthodologiquement hors d'atteinte. Par contre, en milieu fluvial, l'estimation des abondances est, certes complexe, mais néanmoins envisageable par le biais d'une stratégie d'échantillonnage stratifiée dans l'espace, stratégie sur laquelle nous reviendrons plus loin.

Partant de cette estimation, la modélisation peut être envisagée en distinguant deux étapes fondamentales, différentes dans les méthodologies mises en oeuvre comme dans les objectifs qu'elles permettent d'atteindre (Figure 3).

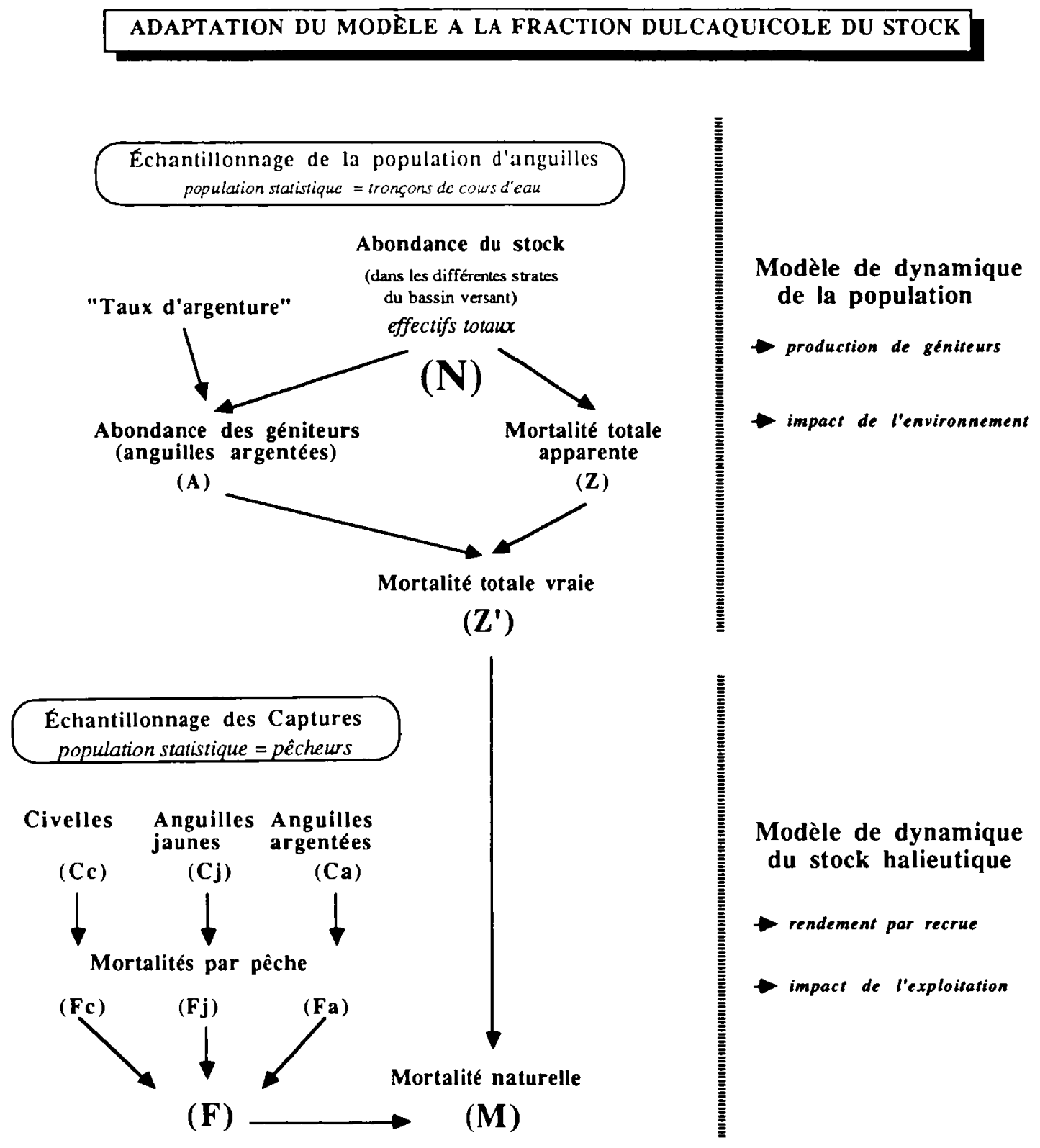

Figure 3 : Schéma illustrant l'adaptation du modèle de rendement par recrue à la fraction dulçaquicole d'un stock d'anguilles dans un bassin versant. Les différentes grandeurs se réfèrent à des matrices par groupes d'âges et par année.

Figure 3 : Flow chart of the adaptation of a yield per recruit model to the freshwater part of an eel stock within a watershed. All different parameters refer to age groups and yearly matrices. 
La première étape concerne la modélisation de la dynamique de la population. De l'estimation des effectifs par année et par groupe d'âge dans différentes strates spatiales, on peut déduire des taux instantanés de Mortalité totale apparente dans chaque strate. L'estimation de "taux d'argenture" doit permettre de quantifier, d'une part, la Mortalité vraie ( $Z$ '), et d'autre part la "Production" de géniteurs (A) (avant Mortalité par pêche et Mortalité naturelle lors de l'avalaison).

Ce modèle atteint d'abord un objectif cognitif. De toute évidence, la connaissance de la production totale par groupe d'âge en Anguilles argentées d'un bassin versant est d'un grand intérêt ; la comparaison de la structure démographique de la population entre strates spatiales différentes est source potentielle d'une meilleure compréhension des migrations à l'intérieur du bassin versant ; les variations spatiales de la "Mortalité vraie" peuvent renseigner de manière déterminante sur l'impact de l'environnement, et tout particulièrement sur l'impact des aménagements réalisés ou des perturbations anthropiques affectant le milieu.

Cette étape de la modélisation peut aussi répondre à un second objectif de gestion du milieu. II devrait par exemple être possible de répondre avec un minimum de certitude à la question suivante : quel serait l'impact, en terme d'abondance globale du stock et de production de géniteurs à l'échelle du bassin versant, d'une diminution de la mortalité naturelle, dans telle strate spatiale où les valeurs de cette mortalité sont anormalement élevées en liaison avec une dégradation de la qualité biologique du milieu (comparaison avec une strate équivalente de bonne qualité biologique) ?

La seconde étape de la modélisation concerne le stock halieutique et passe donc par une prise en compte de l'exploitation tant par les pêcheurs amateurs que par les professionnels. L'estimation des captures par âges, dans les différentes strates spatiales et pour les différents stades biologiques, doit permettre de discriminer au sein de la Mortalité totale ( $\left.Z^{\prime}\right)$ les termes de "Mortalité par pêche" (F) et de "Mortalité naturelle" (M). Notons que l'incertitude liée à l'estimation des captures, n'a pas ici les mêmes conséquences que dans l'analyse des cohortes. La difficulté peut en particulier être contournée par des études de sensibilité et par des techniques de simulation.

Comme la précédente, cette étape de l'analyse constitue un outil explicatif et un outil décisionnel. Elle permet cette fois de quantifier les interactions entre pêcheries, ou les effets respectifs de l'exploitation et d'une dégradation éventuelle du milieu ; elle débouche sur des simulations et des prévisions du rendement par recrue et de la production de géniteurs en fonction des paramètres relatifs à la gestion des pêcheries d'une part et à la gestion des milieux d'autre part.

Dans un premier temps, l'ajustement de ce type de modèle sur les données concernant une année peut conduire à un diagnostic sur l'état du stock. Compte tenu de la variabilité spatiale des phénomènes, celui-ci doit être riche d'enseignements en matière de gestion. A plus long terme, la prise en compte de la variabilité inter-annuelle de la population suppose l'ajustement des modèles sur des données estimées au cours de plusieurs années successives.

\section{ESTIMATION DES PARAMÈTRES}

La possibilité d'estimer les paramètres d'entrée des modèles précédemment présentés est naturellement un préalable à la démarche présentée.

La première difficulté réside dans la nécessité d'une estimation des captures et des effectifs du stock par groupe d'âge. Il faut donc pouvoir déterminer l'âge, et disposer d'une méthode utilisable en routine. Les travaux récents (MOUNAIX, 1992 a et b ; PANFILl et al., 1992) semblent répondre au premier point. En particulier, les études otolithométriques permettent d'attribuer un âge relatif aux individus. II s'agit cependant de méthodes "lourdes" faisant en particulier appel à la microscopie électronique et par conséquent difficilement applicables en routine. Une rapide validation sera nécessaire pour l'application à un bassin versant particulier. Comme c'est très généralement le cas en halieutique, on devra dans un premier temps estimer les captures ou les effectifs du stock par classe de taille, puis convertir les structures de taille en structures démographiques au moyen de clés de conversion taille / âge. L'utilisation de clés, établies à une période donnée et donc considérées constantes, pose de nombreux problèmes notamment liés à la variabilité temporelle de la croissance et de l'abondance des cohortes (KIMURA, 1977). 
Des méthodes de correction sont cependant disponibles en particulier en ce qui concerne la prise en compte de la variabilité d'abondance des cohortes (BARTOO et PARKER, 1983 ; HOENIG et HEISEY, 1987 ; KIMURA et CHICKUNI, 1987 ; GASCUEL, 1993).

La seconde difficulté réside dans l'estimation par échantillonnage des effectifs totaux du stock à l'échelle du bassin versant. La stratégie d'échantillonnage, qui nous paraît la plus appropriée, peut être résumée comme suit :

La population statistique prise en compte est l'ensemble des tronçons de cours d'eau, formant le réseau hydrographique. Chaque tronçon est défini comme un linéaire de cours d'eau, délimité à l'amont et à l'aval par une confluence (ou une dichotomie pour l'aval). A partir d'une analyse cartographique, chaque tronçon peut aussi être caractérisé par sa longueur et par un ensemble de variables descriptives supposées déterminantes dans la structuration d'un peuplement en anguille (ordre hydrographique, distance à la mer, altitude, pente moyenne, substrat géologique,...). Dans un certain nombre de tronçons, tirés par échantillonnage, la densité d'anguilles peut être estimée, en particulier par des opérations de pêche électrique. Ces densités sont exprimées en nombre d'anguilles par $\mathrm{km}$ linéaire et permettent donc une extrapolation à l'ensemble du réseau hydrographique, la longueur totale de celui-ci étant connu.

Au sein de la population statistique des tronçons, une stratification doit être opérée. Des études préliminaires, s'appuyant sur les données actuellement disponibles d'inventaires par pêche électrique (et par d'autres méthodes dont certaines sont encore à mettre au point pour des profondeurs supérieures à un mètre), sont nécessaires pour déterminer des critères de stratifications, c'est-à-dire pour choisir les variables descriptives définissant les strates. Le choix dépend naturellement des relations existant entre variables et densités d'anguilles observées. L'estimation débouche ainsi sur une estimation de l'abondance par strate et totale, ainsi que sur une quantification des variances d'estimation (mesure de l'incertitude). La stratification doit en particulier permettre de prendre en compte la variabilité spatiale du peuplement à l'échelle du bassin versant. On notera que la nécessité de disposer d'une estimation extrapolée à l'ensemble du stock (c'est-à-dire à tout le réseau hydrographique) impose à priori d'écarter de la liste des variables utilisables pour la stratification des paramètres tels que la profondeur, l'habitat ou le faciès. Ces demiers restent cependant d'un intérêt évident pour l'étude de la micro-répartition ou de l'écologie de l'espèce. Ils sont, par contre, peu opérationnels en matière de modélisation de la dynamique car ils ne sont connus en général que pour quelques points de l'espace. En revanche, la variabilité spatiale d'abondance, induite par ces facteurs, peut être considérée, au moins partiellement, par l'intermédiaire des variables précédemment citées.

C'est également au cours de cette procédure d'échantillonnage que doivent être estimés les "taux d'argentures". Ici encore, un travail préliminaire s'impose pour définir des critères éco-physiologiques et /ou morphologiques de "pré-argenture" afin de pouvoir quantifier la proportion des animaux susceptibles de dévaler dans les mois suivants. Un échantillonnage au sein de la population des pêcheurs doit ainsi viser à estimer les captures totales et de là, préciser la répartition en taille de ces captures.

Enfin, concemant l'estimation des captures, une stratégie d'échantillonnage doit également être mise en place en reprenant un schéma de stratification proche de celui présenté dans le cas des effectifs du stock.

\section{DISCUSSION}

L'estimation des paramètres de la modélisation reste relativement complexe et nécessite un travail de recherche mené sur le moyen ou le long terme. Ceci n'est cependant pas une particularité propre à l'Anguille. L'histoire de l'halieutique marine montre en particulier que de premières estimations grossières débouchent sur des diagnostics souvent douteux dans un premier temps mais qui s'affinent progressivement au fur et à mesure d'une acquisition plus fiable des données utilisées (LAUREC et LE GUEN, 1981 ; LAUREC, 1990). Les phénomènes principaux sont évidemment mis en évidence les premiers; la compréhension et la simulation des phénomènes plus complexes ne peuvent être envisagées que par la suite. Par nature, cette approche est très différente de celle, plus classique en écologie, qui consiste à analyser les phénomènes élémentaires avant d'en envisager l'intégration dans un schéma général d'analyse de l'ensemble du système. 
Dans un premier temps, elle doit permettre l'ajustement des modèles de dynamique des stocks dans un ou quelques bassins versants judicieusement choisis (ANONYME, $1984 \mathrm{a}$ ). A plus long terme, la prise en compte de différents bassins versants doit conduire à une vision plus globale du stock d'Anguille à l'échelle nationale, voire européenne. Elle peut ainsi contribuer à l'étude la phase pré-recrutée qui reste naturellement essentielle.

Plus généralement, on doit noter que les modèles de dynamique des populations halieutiques sont rarement utilisés dans le cas des ressources dulçaquicoles. Pour l'Anguille, très peu sont disponibles hormis les travaux de LEE (1979), SPARRE (1979), GATTO et al. (1982), ROSSI et CANNAS (1984), VOLLESTAD et JONSSON (1988), NAISMITH et KNIGHTS (1990), VOLLESTAD (1990).

La première raison de ce manque de références réside sans doute dans l'absence ou la faiblesse des exploitations à caractère commercial en milieu fluvial. Ceci se traduit par l'absence de statistiques de pêches et surtout, compte tenu des enjeux économiques limités, par une recherche scientifique structurellement faible. Cette situation pourrait actuellement évoluer, en liaison notamment avec une revalorisation de la notion de patrimoine naturel et une meilleure prise de conscience de la nécessité de gérer les ressources vivantes renouvelables.

La variabilité du milieu constitue une deuxième difficulté pour la modélisation. L'hypothèse de base de toute la dynamique des populations est que l'exploitation des stocks constitue sinon la seule, en tout cas la principale source de variabilité de l'abondance des stocks. En milieu marin, l'expérience a montré que cette hypothèse est valide pour de nombreux stocks. En milieu dulçaquicole sa validité apparaît beaucoup plus hasardeuse. L'adaptation du modèle que nous avons proposé se fixe cependant précisément pour objectif de résoudre la difficulté tant en terme d'ajustement que d'utilisation du modèle.

Enfin, il faut noter que la méconnaissance relative des méthodes de modélisation par la communauté scientifique concernée constitue sans doute un facteur d'explication de la faiblesse des recherches menées en ce sens en milieu dulçaquicole. De ce point de vue, une approche inter-disciplinaire associant spécialistes de ces milieux et dynamiciens des populations halieutiques reste très largement à mettre en place.

\section{CONCLUSION}

La validation de l'approche conceptuelle présentée ici passe bien évidemment par sa mise en œuvre pratique. De premiers travaux en ce sens ont notamment été engagés au sein du laboratoire Halieutique de l'ENSAR. Ils concernent principalement : les méthodes d'estimation de l'âge ; l'estimation des captures en milieu estuarien et la modélisation de la fraction du stock correspondante ; les méthodes de stratification du réseau hydrographique en vue d'une estimation des effectifs à l'échelle du bassin versant.

Pour l'essentiel, l'application reste cependant à mettre en œuvre. C'est elle qui permettra notamment de déterminer si l'effort d'échantillonnage qu'il est possible de réaliser conduit à des résultats d'une fiabilité suffisante pour qu'ils présentent un caractère opérationnel en terme de gestion. Cette mise en œuvre impliquera une mobilisation des moyens de recherche et une collaboration accrue entre les partenaires concernés au plan national et / ou international. Celleci ne peut se concevoir sans une vision prospective claire des objectifs poursuivis, des méthodes à utiliser, et des résultats qu'on peut en attendre. C'est dans le souci de préciser ces trois aspects que la présente note a été rédigée.

\section{BIBLIOGRAPHIE}

ANONYME, 1984 a. Groupe National Anguille. Rapport de synthèse. Ministère de l'Environnement, Ministère de l'Agriculture, Ministère de la Mer. 60 p. ronéo.

ANONYME, 1984 b. Groupe National Anguille. Rapports thématiques. Ministère de l'Environnement, Ministère de l'Agriculture, Ministère de la Mer. 219 p. ronéo.

ANONYME, 1988. Report of the Working Group on the assessment of the European eel. Conseil International pour l'Exploration de la Mer, C.M. 1988/Assess: 7, 12 p. ronéo. 
BABIN D., 1983. Contribution à l'étude d'une ressource naturelle renouvelable : la pêche professionnelle en eau douce en France. Thèse Doctorat Géographie \& aménagement de l'espace, Université Rennes 2, 212 p, 54 annexes, un atlas.

BARTOO N.W., PARKER K.R., 1983. Stochastic age frequency estimation using the Von Bertalanffy growth equation. U.S. Nat. Mar. Serv., Fish. Bull., 81, 91-96.

BEN ABDALLAH L., 1991. Influence de quelques facteurs abiotiques sur l'abondance de civelles d'Anguilla anguilla L., dans l'estuaire de la Loire, France. Communication Working Group on Eel, CECPI / FAO, Dublin, 21 p. ronéo.

BRETHES J.C., O'BOYLE R.N. (Ed.),1990. Méthodes d'évaluation des stocks halieutiques. Université du Québec, Rimouski, Vol I et II, 963 pages.

COLOMBO G., GRANDI G., ROSSI R., 1984. Gonad differenciation and body growth in Anguilla anguilla L.. J. Fish Biol., 24 (2), 215-228.

CUSHING D.H., 1973. Dependance of recruitment on parent stock. J. Fish. Res. Bd Can., 30 (12), 1965-1976.

DESAUNAY Y., GUERAULT D., BEILLOIS P., 1987. Dynamique de la migration anadrome de la civelle (Anguilla anguilla L.) dans l'estuaire de la Loire : rôle des facteurs climatiques vis-à-vis de la pêche et du recrutement. Conseil International pour l'Exploration de la Mer, Comité des Poissons anadromes et catadromes, C.M. 1987/M: 18, 22 p. ronéo.

ELIE P., RIGAUD C., 1984. Etude de la population d'anguilles de l'estuaire et du bassin versant de la Vilaine : pêche, biologie, écologie. Examen particulier de l'impact du barrage d'Arzal sur la migration anadrome (civelles). Rapport contrat Union Régionale des Fédérations d'Associations Agréées de Pêche et de Pisciculture / Université de Rennes 1, $120 \mathrm{p}$.

ELIE P., ROCHARD E., BOIGONTIER B., 1989. Etude de suivi halieutique de l'estuaire de la Gironde en 1988. Rapport CEMAGREF de Bordeaux, Division ALA / EDF, 169 pages.

GASCUEL D., 1987 a. Captures, CPUE, abondance et dynamiques de migration des civelles (Anguilla anguilla $L$., ) dans l'estuaire de la Sèvre Niortaise : 1961-1983. Communication Working Group on Eel, CECPI / FAO, Bristol, 12 p. ronéo.

GASCUEL D., 1987 b. La civelle d'anguille dans l'estuaire de la Sèvre Niortaise : biologie, écologie, exploitation. Publications Département Halieutique, Ecole Nationale Supérieure Agronomique, Rennes, 4 (1), $355 \mathrm{p}$.

GASCUEL D., 1993. Une méthode simple d'ajustement des clés "taille - âge": application aux captures d'albacores (Thunnus albacares) de l'Atlantique Est. Can. J. Fish Aquat. Sci. (sous presse).

GASCUEL D., FONTENELLE G., 1989. Caractéristiques biologiques d'un peuplement estuarien d'anguilles sub-adultes (estuaire de la Sèvre Niortaise, France). Communication Working Group on Eel, CECPI/FAO, Porto, 12 p. ronéo.

GASCUEL D., FONTENELLE G., 1990. Biological characteristics of an estuarine growing Eel population (Sèvre Niortaise estuary, France). Int. Revue ges. Hydrobiol., 75 (6), 796.

GATTO M., LANIADO E., ROSSI R., 1982. The management of eels in the valli di Comacchio lagoon. Oceanol. Acta, 303-307.

GUERAULT D., BEILLOIS P., DESAUNAY Y., DOREL D., 1986. Variations de l'abondance de la civelle au travers des données de production : secteurs Loire et Vilaine. Vie et Milieu, 36 (4), 237-242.

GUERAULT D., BEILLOIS P., DESAUNAY Y., 1987. L'exploitation de la civelle (Anguilla anguilla L.) en Loire et Vilaine : indices d'abondance et indicateurs halieutiques. Conseil International pour l'Exploration de la Mer, Comité des Poissons anadromes et catadromes, C.M. 1987/M : 19, 22 p. ronéo. 
GUERAULT D., DESAUNAYY., 1989. Evolution de l'abondance de la civelle (Anguilla anguilla L.) dans les estuaires de la Loire et de la Vilaine (France) : 1977-1988. Communication Working Group on Eel, CECPI/FAO, Porto, 20 p. ronéo.

GUERAULT D., LECOMTE R., DESAUNAY Y., BIAGIANTI S., BEILLOIS P., GRELLIER P., 1991. The glass-eel arrivals in the Vilaine estuary (Northern Biscay Bay) through the year 1990 : demographic features and early life history. Communication Working Group on Eel, CECPI/FAO, Dublin (Irlande), 28 p. ronéo.

GULLAND J.A., 1965. Estimation of mortality rates. Annex to Arctic Fisheries Working Group Report. Intern. Counc. Explor. Sea, CM 1965, Doc n 3. ronéo.

HOENIG J.M., HEISEY D.M., 1987. Use of a log-linear model with the EM algorithm to correct estimate of stock composition on to convert length to age. Trans. Amer. Fish. Soc., 116, 232-243.

KIMURA D.K., 1977. Statistical assessment of the age-length key. J. Fish. Res. Bd Can., 34 (4), 317-324.

KIMURA D.K., CHICKUNI S., 1987. Mixture of empirical distribution : an iterative application of the age-length key. Biometrics, 43, 23-35.

LAUREC A., 1990. Les outils de gestion des stocks : performances, limites, évolution. In GASCUEL D. (Ed.), "Rencontres halieutiques de Rennes" - La gestion des pêches maritimes françaises. Actes du Colloque, 11-18, Ecole Nationale Supérieure Agronomique, Rennes.

LAUREC A. et LE GUEN J.C., 1981. Dynamiques des populations marines exploitées. Tome 1 : Concepts et modèles. Rapports scientifiques et Techniques CNEXO, 45, 118 p.

LEE T.W., 1979. Dynamique des populations d'anguilles (Anguilla anguilla L.) des lagunes du bassin d'Arcachon., Thèse Doctorat Ecologie générale \& appliquée, option Ecologie aquatique. Université Sciences \& Techniques Languedoc, Montpellier, 218 p.

LEGAULT A., 1986. Distribution du peuplement d'anguilles (Anguilla anguilla L.) à l'échelle d'un bassin versant. Vie et Milieu, 36 (4), 261-265.

LEGAULT A., PORCHER J.P., 1989. Distribution du peuplement d'anguilles (Anguilla anguilla L.) à l'échelle régionale Bretagne-Basse-Normandie, France. EIFAC Working Party on Eel, Porto, $12 \mathrm{p}$.

MOUNAIX B., 1991. Utilisation des otolithes pour caractériser l'habitat de l'anguille jaune dans le bassin de la Vilaine, Bretagne. EIFAC Working Party on Eel, Dublin (Irlande), 11 p. ronéo.

MOUNAIX B., 1992 a. Intercalibration et validation des méthodes d'estimation de l'âge de l'Anguille européenne (Anguilla anguilla, L.). Application au bassin versant de la Vilaine, Bretagne. Thèse Doctorat Halieutique, Publications du Département Halieutique, 14, Ecole Nationale Supérieure Agronomique, Rennes, $146 \mathrm{p}$.

MOUNAIX B., 1992 b. Validation de l'estimation de l'âge de l'Anguille européenne (Anguilla anguilla L.) dans le bassin versant de la Vilaine, Bretagne : résultats préliminaires. In BAGLINIERE J.L., CASTANET J., CONNAND F. et MEUNIER F.J. (Ed.), Tissus durs et âge individuel des Vertébrés, 104-118, ORSTOM-INRA, Paris.

NAISMITH I.A., KNIGHTS B., 1990. Modelling of unexploited and exploited populations of eels, Anguilla anguilla (L.), in the Thames estuary. J. Fish Biol., 37, 975-986.

O'BOYLE R.N., 1990. Estimation de l'abondance et de la mortalité par pêche par l'analyse séquentielle des populations. In BRETHES J.C., O'BOYLE R.N. (Ed.), Méthodes d'évaluation des stocks halieutiques, 465-507, Université du Québec, Rimouski.

PANFILI J., XIMENES M.C., CRIVELLI A., DO CHI T., 1992. Validation de l'âge de l'Anguille: résultats préliminaires en Méditerranée. In BAGLINIERE J.L., CASTANET J., CONNAND F. et MEUNIER F.J. (Ed.), Tissus durs et âge individuel des Vertébrés, 119-128, ORSTOMINRA, Paris. 
PAULICK G.J., BAYLIFF W.H., 1967. A generalized computer program for the Ricker model of equilibrium yield per recruitment. J. Fish. Res. Bd Can., 11 (2), 249-259.

POPE J.G., 1972. An investigation of the accuracy of virtual population analysis using cohort analysis. ICNAF Res. Bull., 9, 65-74.

POPE J.G., SHEPHERD J.G., 1985. A comparison of the performance of various methods for tuning VPAs using effort data. J. Cons. int. Explor. Mer, 42, 129-151.

RICKER W.E., 1945. A method of estimating minimum size limits for obtaining maximum yield. Copeia, 1945 (2), 84-94.

RICKER W.E., 1958. Handbook of computations for biological statistics of fish populations. Bull. Fish. Res. Bd Can., 119, 300 p.

ROSSI R., CANNAS A., 1984. Eel fishing management in a hypersaline lagoon of southern Sardinia. Fish. Res., 2, 285-298.

ROSSI R., COLOMBO G., 1979. Some observations on age, sex and growth of silver eels (Anguilla anguilla L.) in north Adriatic lagoons. Rapp. P.-v. Réun. Cons. int. Explor. Mer, 174, 64-69

SPARRE P., 1979. Some necessary adjustements for using the common methods in eel assessment. Rapp. P.-V. Cons. int. Explor. Mer, 174, 41-44.

TESCH F.W., 1980. Occurrence of eel (Anguilla anguilla L.) larvae west of the European continental shelf : 1971-1977. Env. Biol. Fish, 5, 185-190.

VOLLESTAD L.A., 1989. Effect of growth rate on age at maturity of the European eel (Anguilla anguilla L.) Communication Working Group on Eel, CECPI/FAO, Porto, 22 p. ronéo.

VOLLESTAD L.A., 1990. Optimal management of European eel in the Imsa River, Norway. J. Conseil, 46 (2), 115-120.

VOLLESTAD L.A., JONSSON B., 1988. A 13-year study of the population dynamics and growth of the European eel Anguilla anguilla in a Norwegian river : evidence for density-dependent mortality and development of a model for predicting yield. J. Anim. Ecol., 57, 983-997.

WALTERS C.J., 1969. A generalized computer model for fish population studies. Trans. Amer. Fish. Soc., 98, 505-512. 